\title{
Continuous Processing and Efficient In Situ Reaction Monitoring of Hypervalent Iodine (III) Mediated Cyclopropanation Using Benchtop NMR Spectroscopy
}

\author{
Batool Ahmed-Omer ${ }^{*}{ }^{\dagger}$, Eric Sliwinski ${ }^{\dagger}$, John P. Cerroti ${ }^{\ddagger}$, and Steven V. Ley ${ }^{\dagger}$ \\ ${ }^{\dagger}$ Department of Chemistry, University of Cambridge, Lensfield Road, Cambridge CB2 1EW, \\ U.K. \\ * Magritek GmbH, Gebäude VO (Building VO), Triwo Technopark Aachen, Philipsstrasse 8, \\ 52068 Aachen, Germany.
}

\section{SUPPORTING INFORMATION}

\section{Spinsolve Benchtop NMR Spectroscopy:}

Inline and online monitoring carried with benchtop Spinsolve ${ }^{\mathbb{O}} \mathrm{NMR}^{1}$ :

- ${ }^{1} \mathrm{H}$ probe with detection using a solenoid coil at a frequency of $43.62 \mathrm{MHz}$

- Magnet temperature of $29^{\circ} \mathrm{C}$

- Resolution at $50 \%$ peak height: linewidth $<0.5 \mathrm{~Hz}(12 \mathrm{ppb})$; at $0.55 \%$ peak height linewidth $<$ $20 \mathrm{~Hz}$

- Proton sensitivity of $>100: 1$ for $1 \mathrm{wt} . \%$ ethylbenzene in a single scan

- Fast automatic hardware lock using an external lock configuration (allows use of nondeuterated solvent)

- Automated shimming using a $10 \% \mathrm{H}_{2} \mathrm{O}$ in $\mathrm{D}_{2} \mathrm{O}$ mixture.

- NMR data collected automatically with Spinsolve software and processed automatically using $\mathrm{MNOVA}^{\oplus}$ software.

NMR Experimental Protocols:

- 1D Proton and Proton+ experiment protocol for online monitoring: records spectra using a single 90 degree excitation pulse, covering range from 63 to $-54 \mathrm{ppm}$.

- Reaction Monitoring (RM) protocol, for inline experiment, capture sequence of 1D proton single scans at user selected time intervals. Processing data as stacked spectra in MNOVA.

Pulse sequence parameters for 1D Proton, 1D Proton+ and RM:

- Acquisition bandwidth: $5 \mathrm{kHz}$ (117 ppm)

- $90^{\circ}$ pulse width: 7 microseconds

- Dwell time: 200 microseconds 
- Number of scans: $1,4,16,64,256$ or until stopped

- Acquisition time: $0.4,0.8,1.6,3.2$ or 6.4 seconds

- 2D Quickcosy records a two-dimensional COSY NMR spectrum (1 scan). Pulse sequence parameters:

- Number of points along $\mathrm{t}_{1}: 256$

- $\mathrm{t}_{1}$ increment: $1.43 \mathrm{~ms}$

- Number of points along $t_{2}: 8,192$

- $\mathrm{f}_{1}$ bandwidth: $0.7 \mathrm{kHz}(16 \mathrm{ppm})$

- $\mathrm{f}_{2}$ bandwidth: $5 \mathrm{kHz}(117 \mathrm{ppm})$

- Acquisition time: 1.638 seconds

- 2D JRES records a two-dimensional homonuclear j-resolved spectrum (4 scans). Pulse sequence parameters:

- Number of points along $\mathrm{t}_{1}: 256$

- $\mathrm{t}_{1}$ increment: $5 \mathrm{~ms}$

- Number of points along $t_{2}: 8,192$

- $\mathrm{f}_{1}$ bandwidth: $200 \mathrm{~Hz}$

- $\mathrm{f}_{2}$ bandwidth: $5 \mathrm{kHz}$ (117 ppm)

- Acquisition time: 1.638 seconds

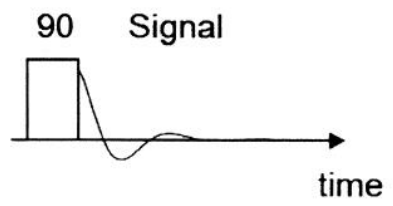

1D Proton pulse sequence.

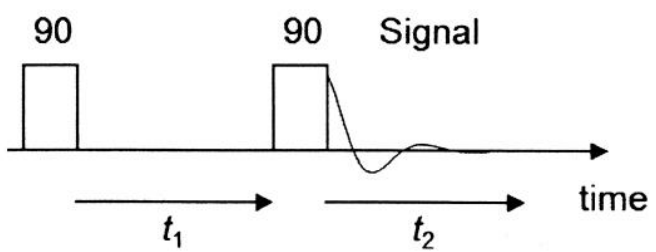

The 2D COSY pulse sequence.

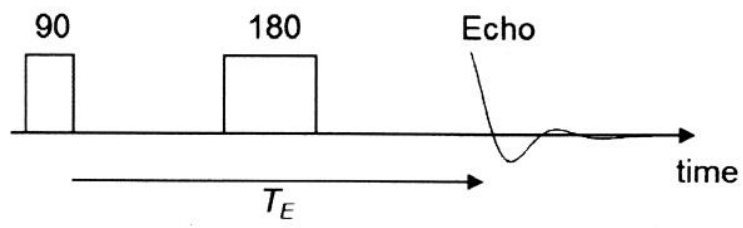

The homonuclear JRES pulse sequence.

Figure S1. Spinsolve ${ }^{1} \mathrm{H}$ NMR pulse sequences. 

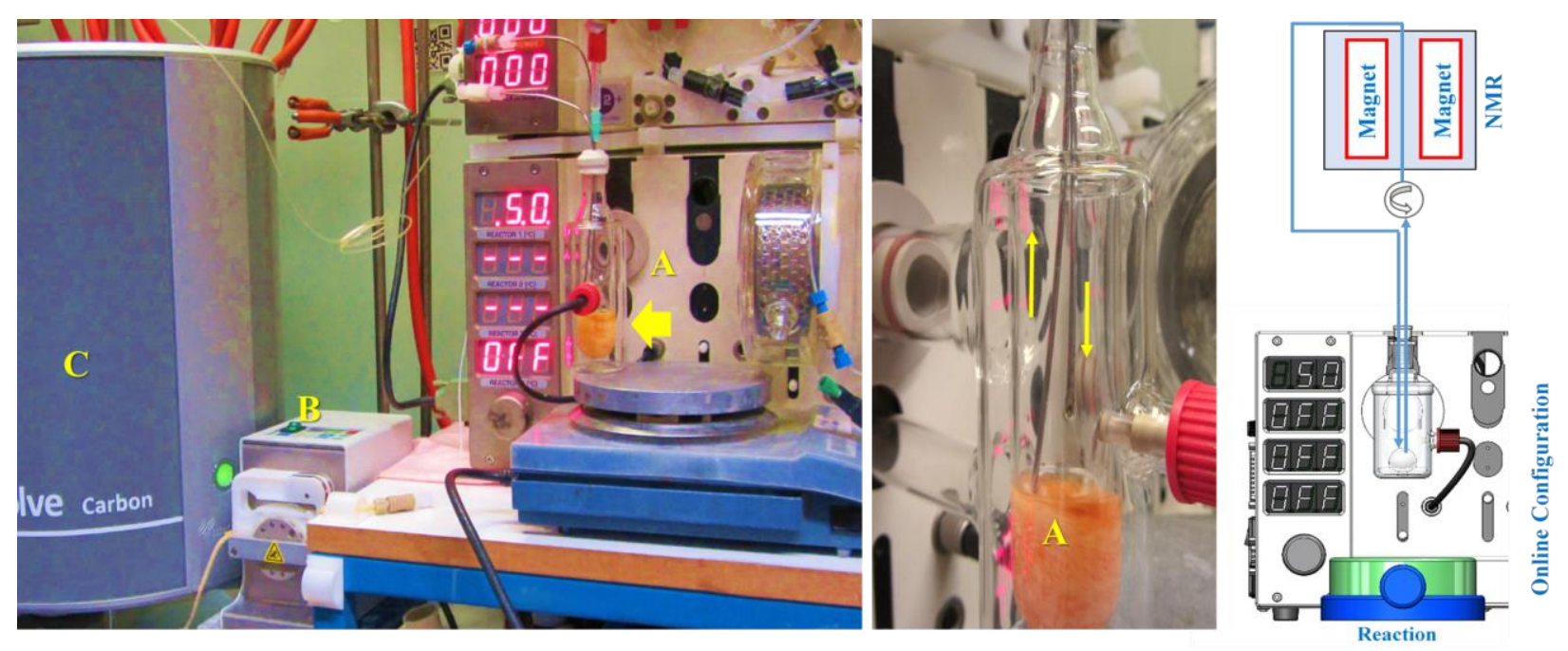

Figure S2. Online reaction monitoring using Spinsolve benchtop NMR integrated with the R4 glass reactor. Legend: (A) R4 glass reactor unit housed on a Vapourtec R4 unit. (B) Digital peristaltic pump (C) Benchtop NMR. (Extra information provided in the supplementary section). 


\section{In-house designed and build R4 Glass Reactor:}

Connected to the Vapourtec ${ }^{\odot} \mathrm{R} 4$ unit and used for online reaction monitoring.

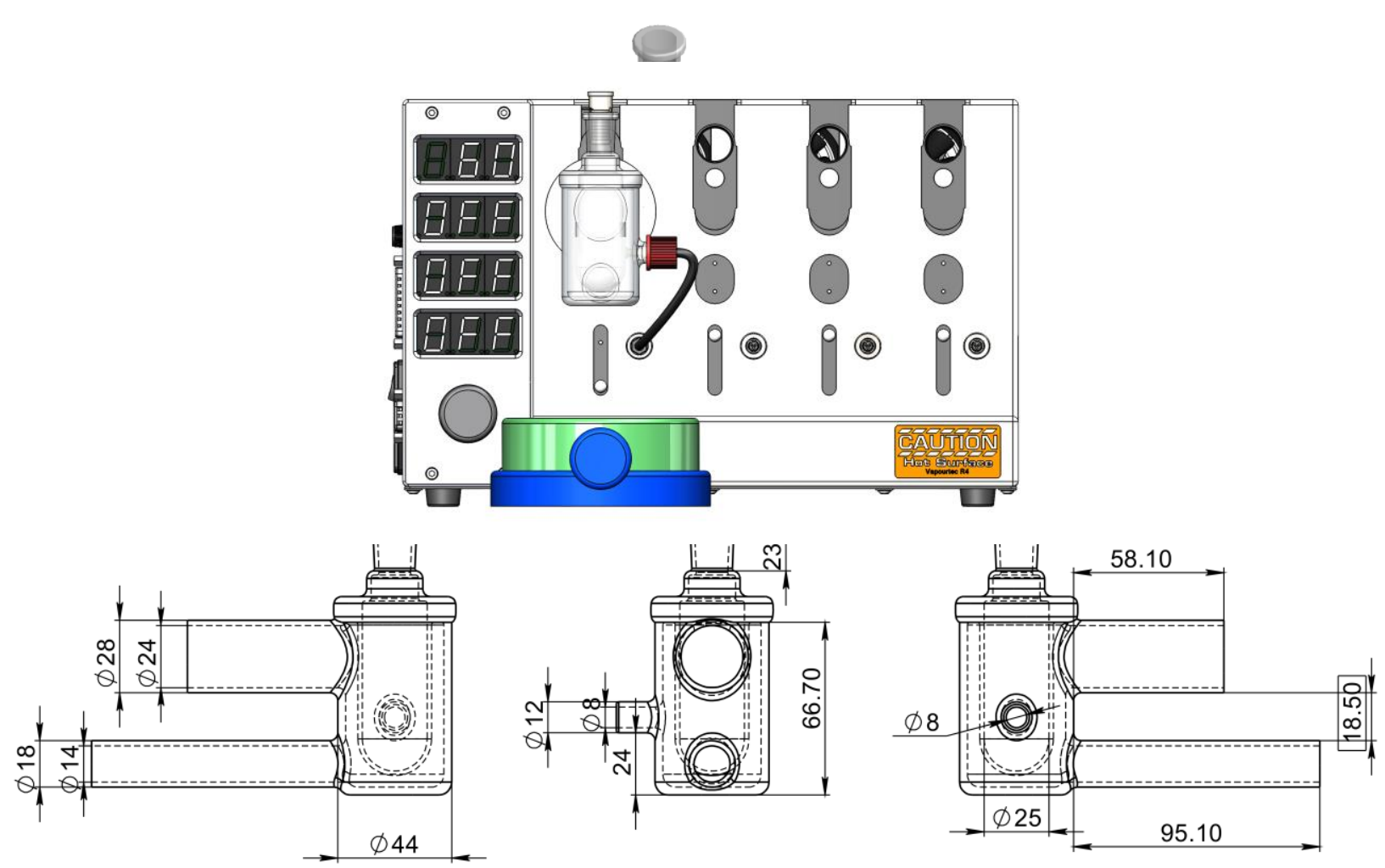

Figure S3. Dimensions of the R4 Glass Reactor.

Figure S4. R4 glass reactor connected to the Vapourtec R4 unit.

\section{Construction of the PTFE Evaporator Prototype:}

The prototype was constructed by the mechanical work-shop at University of Cambridge Department of Chemistry using PTFE (Teflon $\left.{ }^{\circledR}\right)$. The finished parts were thoroughly washed with hexane, soap, water and acetone before assembly (Figure S5). Other components were sourced from Upchurch Scientific, Vapourtec, Omnifit, Cole-Parmer and Hooper as detailed in Table S1. ${ }^{2,3,4,5,6}$

Table S1. Component part list for the PTFE prototype.

\begin{tabular}{cccc}
\hline Label & Supplier & Part Reference & Part description \\
\hline $\mathbf{1}$ & Mechanical & NA & MK2 Evaporator top end piece (PTFE) \\
\hline
\end{tabular}




\begin{tabular}{|c|c|c|c|}
\hline & workshop & & \\
\hline 2 & $\begin{array}{l}\text { Mechanical } \\
\text { workshop }\end{array}$ & NA & MK2 Evaporator connector (PTFE) \\
\hline 3 & Hooper & BS806 & Fluoroelastomer (Viton $\left.{ }^{\circledR}\right)$ O-ring (14 mm o.d., 11.11 mm i.d.) \\
\hline 4 & Cole-Parmer & $06385-05$ & Thread to compression adapters, $1 / 8 " \mathrm{BSP}-\mathrm{P}(\mathrm{M})$ to $1 / 8 "$ \\
\hline 5 & Cole-Parmer & UY-06605-27 & $\begin{array}{l}\text { Flanged PTFE tubing } 1 / 8 \text { inch }(3.175 \mathrm{~mm}) \text { o.d. X 1/16 inch (1.58 } \\
\text { mm) i.d. }\end{array}$ \\
\hline 6 & NA & NA & Galvanised steel spacer \\
\hline 7 & Upchurch & $\mathrm{P} 250 \mathrm{X}$ & 1/4-28 Fitting with super flangeless ferrule \\
\hline 8 & Upchurch & 1535 & $\begin{array}{c}\text { PEEK Tubing, } 1 / 16 \text { inch }(1.587 \mathrm{~mm}) \text { o.d. X 1/200 inch }(0.125 \mathrm{~mm}) \\
\text { i.d. }\end{array}$ \\
\hline 9 & Upchurch & P-580 & Stainless steel ferrule for $1 / 8$ inch tubing flanged fitting \\
\hline 10 & Vapourtec & NA & PTFE column end fitting \\
\hline 11 & Omnifit & $\begin{array}{c}\text { 006BCC-15-10- } \\
\text { FF }\end{array}$ & Column bottom end piece assembly with connection cap. \\
\hline 12 & Omnifit & $\begin{array}{l}\text { 006BCC-15-10- } \\
\text { FF }\end{array}$ & 100 X 15 mm o.d. glass column \\
\hline
\end{tabular}

NB: Part 5 was flanged by contact of a heated piece of 1/8 inch PTFE tubing on a cold orthogonal surface. Part 6, the spacer component was constructed as a 2 active coils, closed, unsquared, not ground compression spring from 4 turns of a $0.80 \mathrm{~mm}$ diameter galvanized steel wire (obtained from a standard paper clip) around $1 \mathrm{~cm}$ of a $1 / 8$ inch diameter stainless steel tubing. 

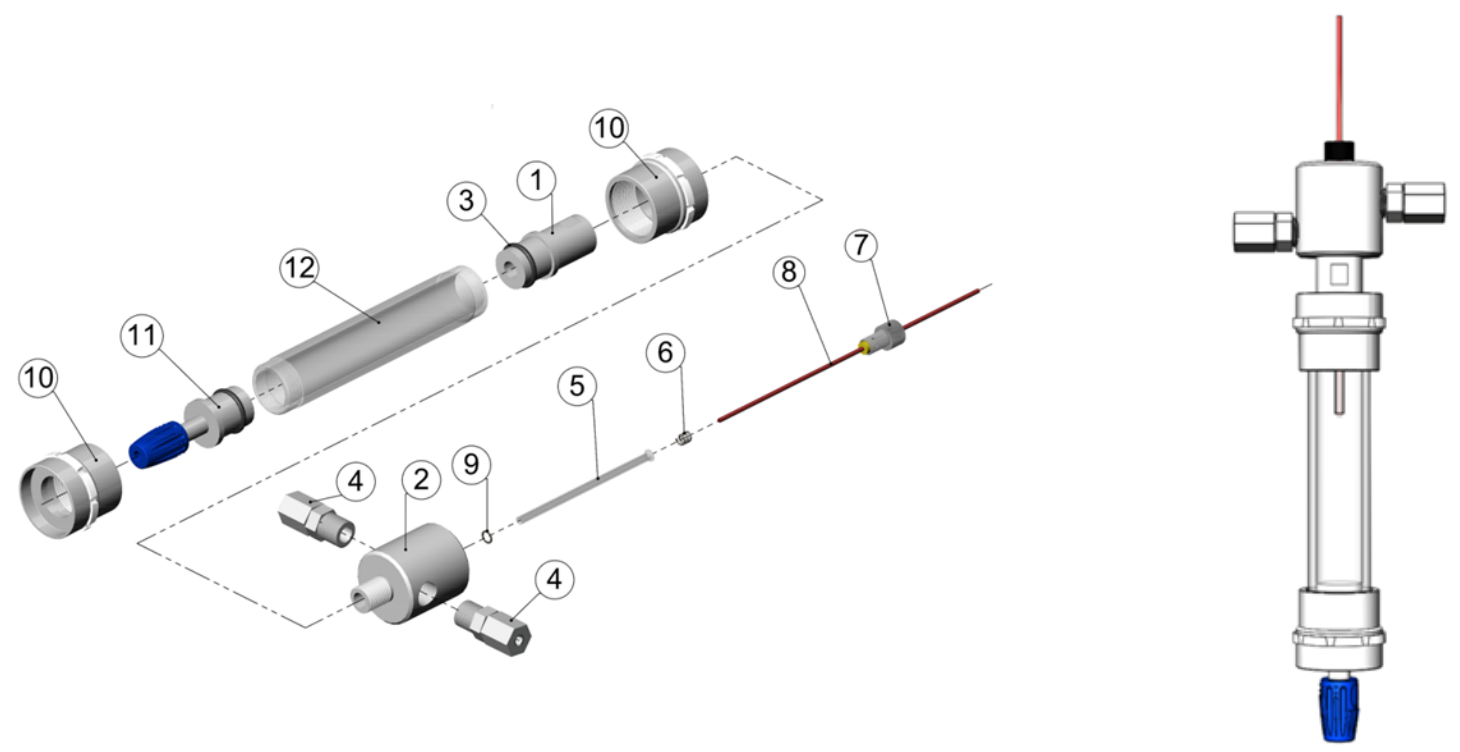

Figure S5. Expanded (left) and assembled (right) diagrams of the evaporator prototype.

The spray tip of the prototype was set as shown in Figure S6. The central 1/16" diameter PEEK tube protruded $1 \mathrm{~mm}$ from the $66 \mathrm{~mm}$ long $1 / 8$ " gas supply tube and $72 \mathrm{~mm}$ from the end of the 1/4-28 fitting.
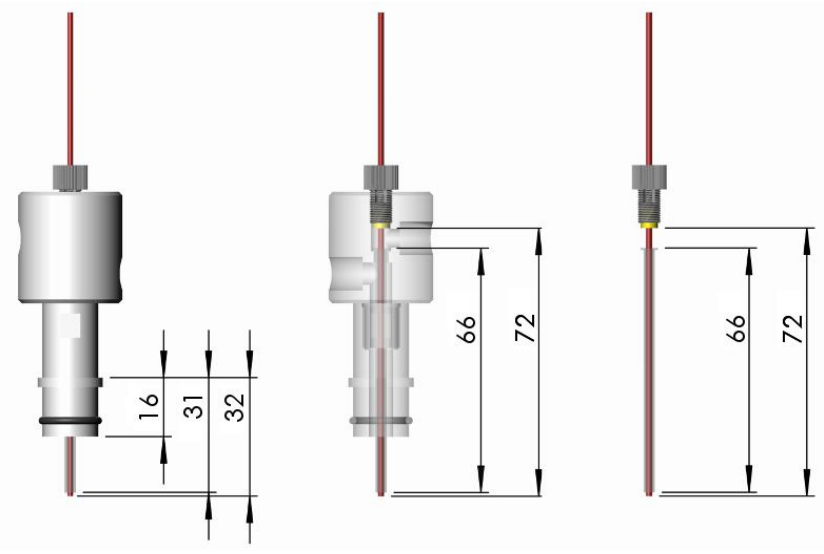

Figure S6. Evaporator prototype spray tip (dimensions are reported in $\mathrm{mm}$ ). 


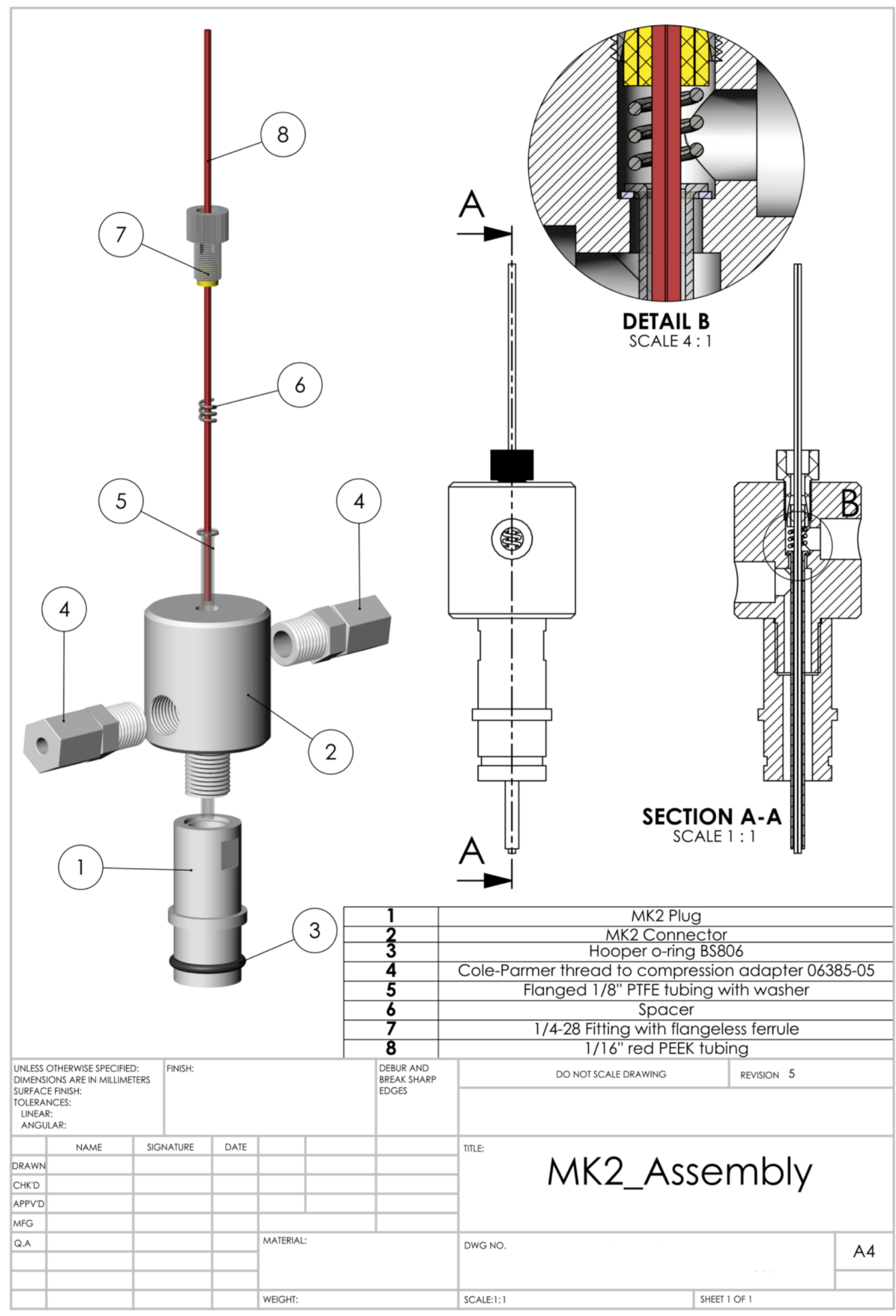




\section{General Operation of Evaporator Prototype:}

The liquid and gas inlets, and the gas/ vapour outlets are shown in Figure S7. The liquid feed to the prototype was pumped by one of the HPLC pumps of a Vapourtec R2+ flow reactor while heating of the evaporators chamber was achieved by placing the Omnifit column inside a glass Vapourtec column heater attached to the Vapourtec R4 flow reactor. ${ }^{3}$ The desolvation gas inlet was connected to a 1 bar in-house compressed air supply. The flow rate of the air stream was set via an analogue adjustable gas flow meter (Cole-Parmer, Ref: 32044-80). The gas outflow of the prototype was connected to a cold trap kept at $-78{ }^{\circ} \mathrm{C}$ by dry ice and acetone, or any other efficient condensing apparatus. The solvent output from the prototype was controlled by a precalibrated Reglo® digital peristaltic pump.
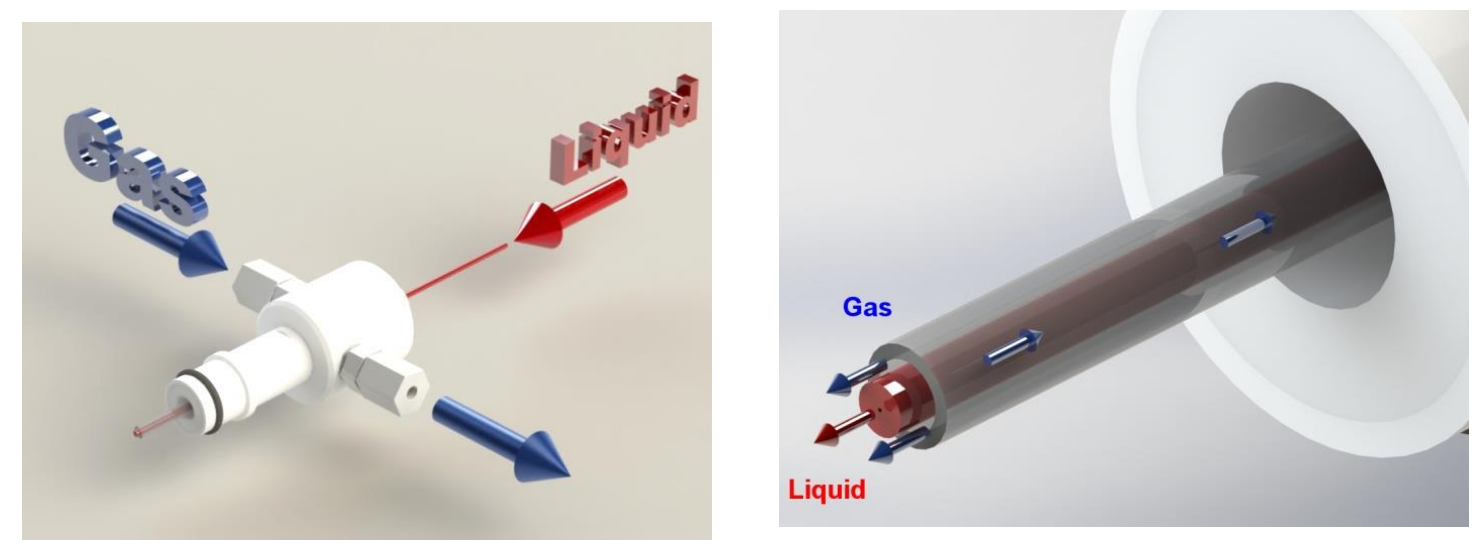

Figure S7. (Left) Evaporator operation. (right) Details of the concentric pneumatic sprayer.

\section{General Procedure for Solvent Switching:}

The prototype was setup as shown in Figure S8. The solvent switching was initially tested using DCM: $\mathrm{CHCl}_{3}$ (Table S2). DCM was pumped into the device by a Vapourtec R2+ pump and the flow rate of the desolvation air stream was maintained accordingly. The evaporation chamber (an Omnifit column) was heated using the Vapourtec R4 column heater. The output of unevaporated solvent was controlled by a pre-calibrated Reglo ${ }^{\circledR}$ digital peristaltic pump. 


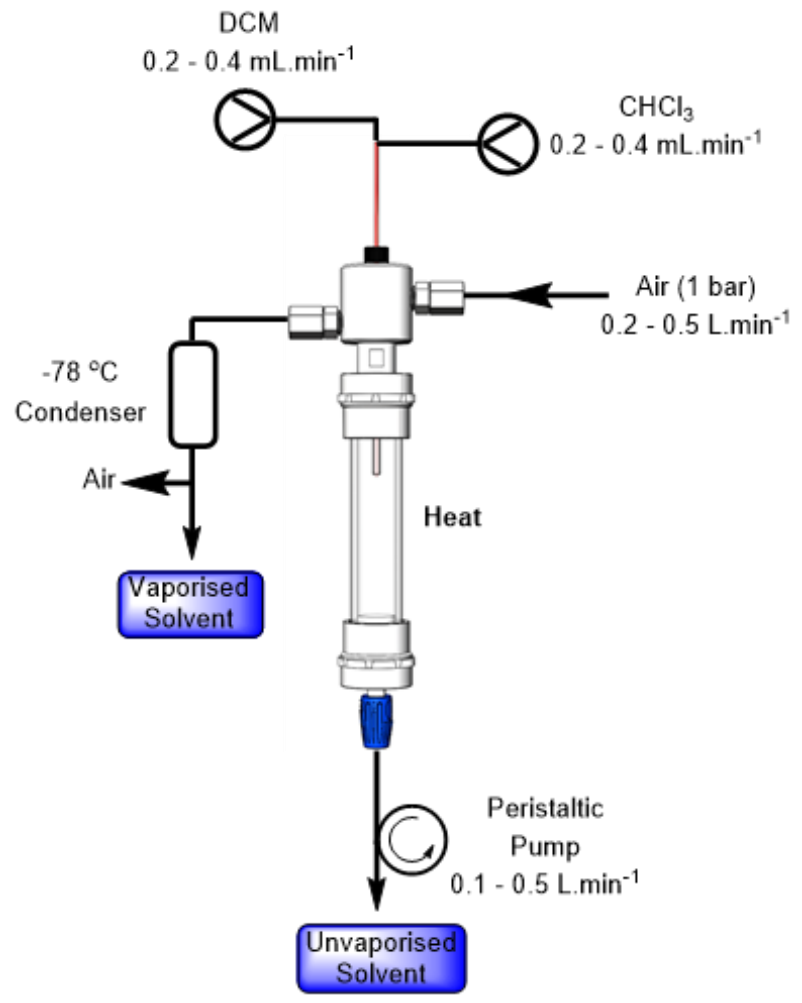

Figure S8. Graphical representation of evaporator setup for solvent switching.

Table S2: Optimization of DCM removal and replacing with $\mathrm{CHCl}_{3}$.

\begin{tabular}{|c|c|c|c|c|c|c|}
\hline Run & $\begin{array}{c}\text { DCM } \\
\left(\mathrm{mL} \cdot \mathrm{min}^{-1}\right)\end{array}$ & $\underset{\left(\mathrm{mL} \cdot \mathrm{min}^{-1}\right)}{\mathrm{CHCl}_{3}}$ & $\begin{array}{c}\mathrm{CHCl}_{3} \\
(\mathrm{~mL})\end{array}$ & $\begin{array}{c}\text { Gas } \\
\left(\text { L. } \text { min }^{-1}\right)\end{array}$ & Temp $^{\circ} \mathrm{C}$ & $\begin{array}{c}\text { Remaining } \\
\text { DCM } \\
(\%)\end{array}$ \\
\hline $1^{\mathrm{a}}$ & 0.4 & 0.4 & 4 & 0.53 & ambient & 3 \\
\hline $2^{b}$ & 0.4 & 0.4 & 4 & 0.32 & ambient & 48 \\
\hline $3^{\mathrm{b}}$ & 0.2 & 0.2 & 4 & 0.32 & ambient & 38 \\
\hline $4^{\mathrm{b}}$ & 0.2 & 0.2 & 4 & 0.32 & 39 & 35 \\
\hline $5^{\mathrm{b}}$ & 0.2 & 0.2 & 4 & 0.32 & 50 & 35 \\
\hline
\end{tabular}

(a) Protocol 1: DCM evaporation for 10 min at specified air flow rate, followed by pumping $\mathrm{CHCl}_{3}$ : ${ }^{\text {(b) }}$ Protocol 2: DCM evaporation for 10 min at specified air flow rate, while pumping $\mathrm{CHCl}_{3}$ 


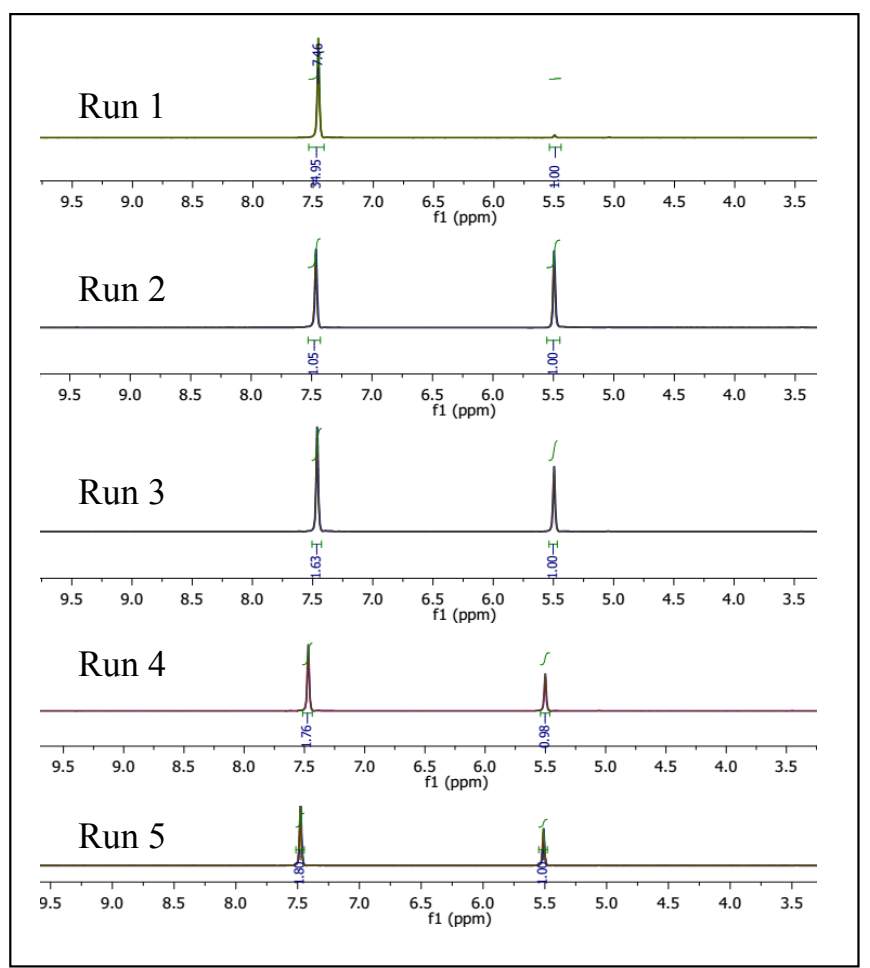

Figure S9. ${ }^{1} \mathrm{H}$ NMR monitoring of $\mathrm{CHCl}_{3}$ peak vs. DCM during optimization using conditions in table S2.
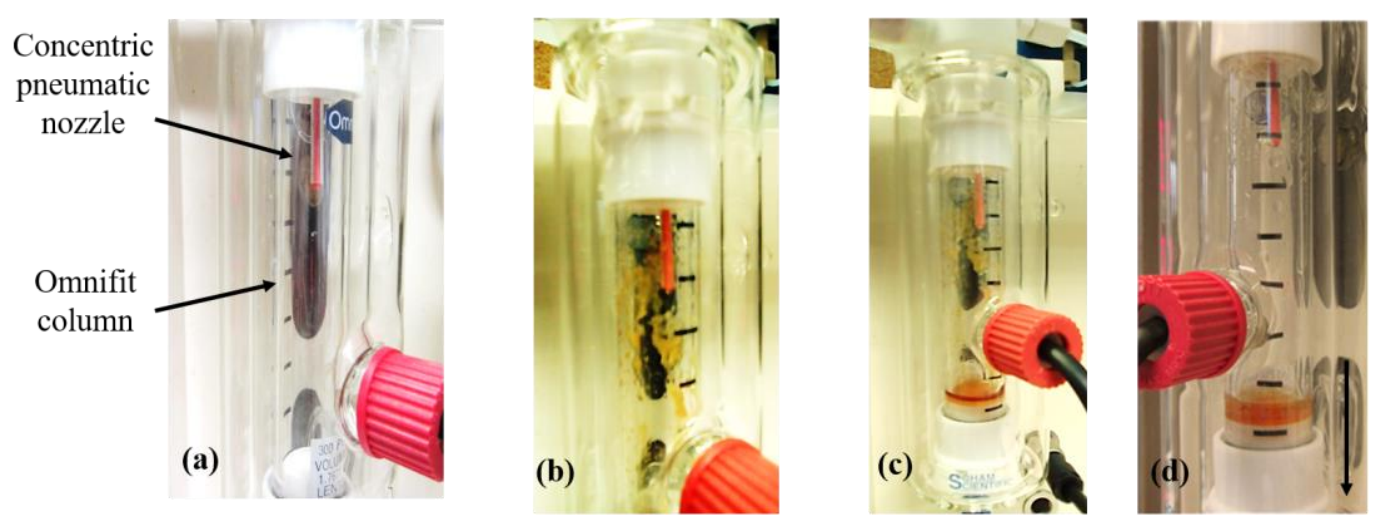

To NMR analysis

Figure S10. Evaporation device in action: (a) Solvent switching device (before spraying) consists of an evaporation chamber (Omnifit column) and a sprayer nozzle, fitted into Vapourtec R4 unit. (b) Evaporation process of reaction flow stream. (c) Washing process of concentrated reaction crude deposited on the Omnifit column using deuterated chloroform.(d) Washing of reaction crude complete and ready to output to NMR analysis using peristaltic pump. 
Table S3: List of selected resonances chosen for the online monitoring of cyclopropanation progress of 4 .

\begin{tabular}{cccccc}
\hline Peak No. & $\boldsymbol{\delta}_{\mathbf{H}}(\mathbf{p p m})$ & Multiplicity & No. Protons & Compound & Moiety \\
\hline $\mathrm{Pk} 1$ & $0.5-1.1$ & $\mathrm{t}$ & $3 \mathrm{H}$ & 5 & $\mathrm{CH}_{3}$-Ethyl \\
$\mathrm{Pk} 2$ & $2.0-2.1$ & $\mathrm{~s}$ & $6 \mathrm{H}$ & $\mathrm{DIB}$ & $\mathrm{OAc}$ \\
$\mathrm{Pk} 3$ & $5.1-5.4$ & $\mathrm{~s} \& \mathrm{dd}$ & $2 \mathrm{H}+1 \mathrm{H}$ & 4 and 2 & $-\mathrm{CH}$ - methylene \\
$\mathrm{Pk} 4$ & $5.5-6.1$ & $\mathrm{dd}$ & $1 \mathrm{H}$ & 2 & $\begin{array}{c}\mathrm{C} \mathrm{e}^{\mathrm{e}} \text {-styrene } \\
\mathrm{CH}^{\mathrm{f}} \text {-styrene }\end{array}$ \\
\hline
\end{tabular}

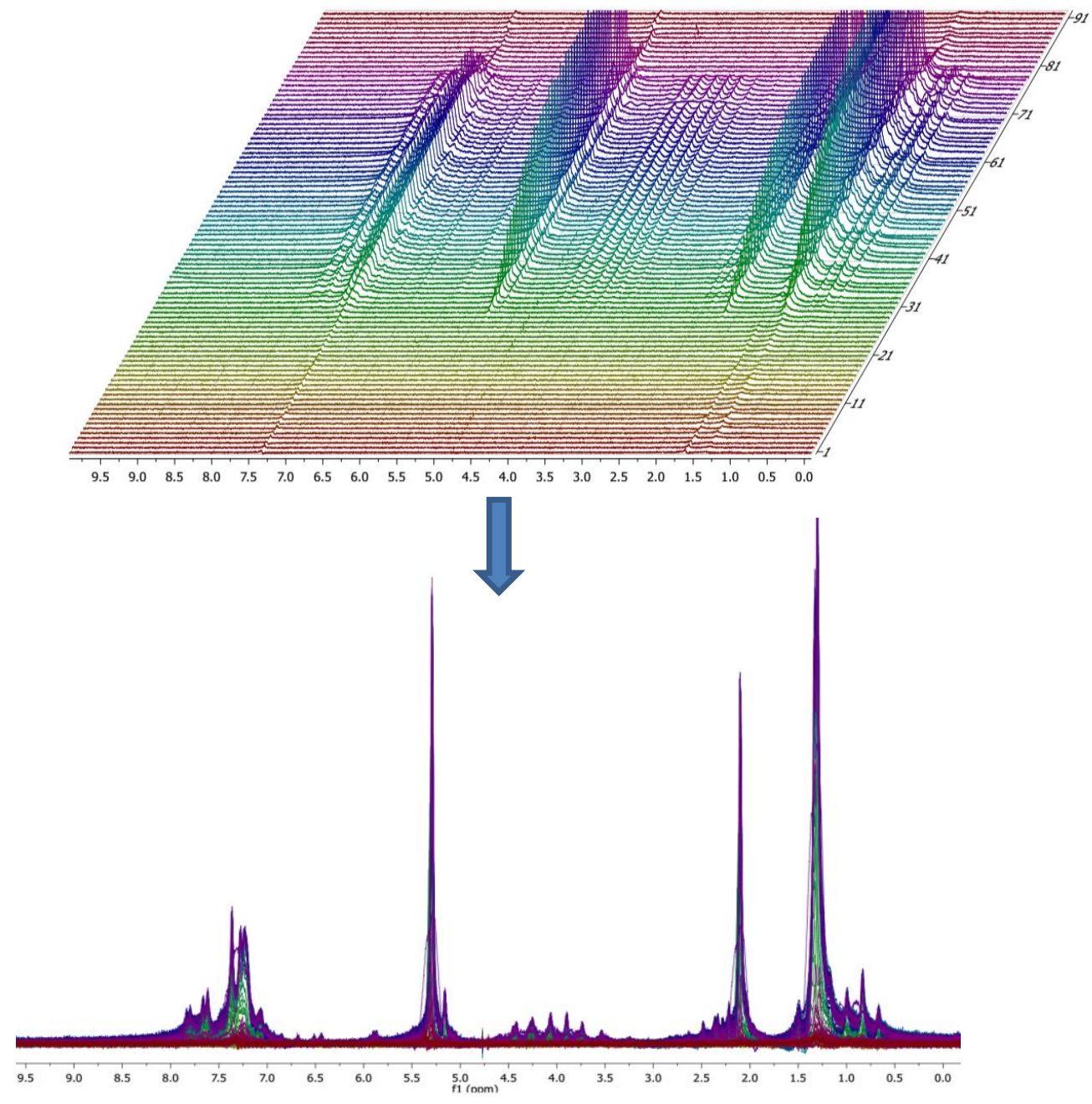

Figure S11. ${ }^{1} \mathrm{H}$ NMR data as array (top) and stacked (bottom), acquired by the inline monitoring of the steady-state of the cyclopropanation of Ethyl-nitroacetate $\mathbf{4}$ under continuous flow. 
TimestampFolder

ProtonPhase

\# Loop

loop(3, 00:13:00:000)

RunProtocol("1D EXTENDED+", ["Number=4", "RepetitionTime=10",

"PulseAngle=90", "AcquisitionTime=6.4"])

wait (00:00:10:000)

RunProtocol("COSY 2D",

cosY-Quick\" ",

"Processing=("MNOVA $\backslash "]$ )

endloop

wait (00:00:10:000)

RunMnovaFile("ScriptUtilities/ReactionMonitor.qs", "process", ["1H"])

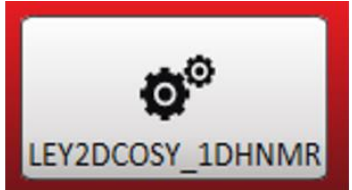

DataFolder
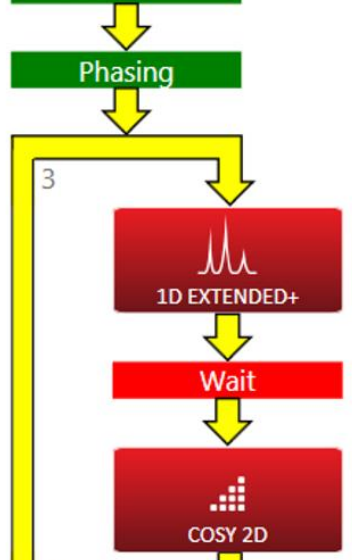

00:13:00:000

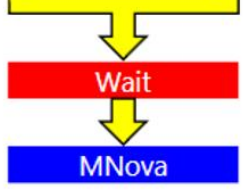

Figure S12. LEY-2D-COSY Script. 


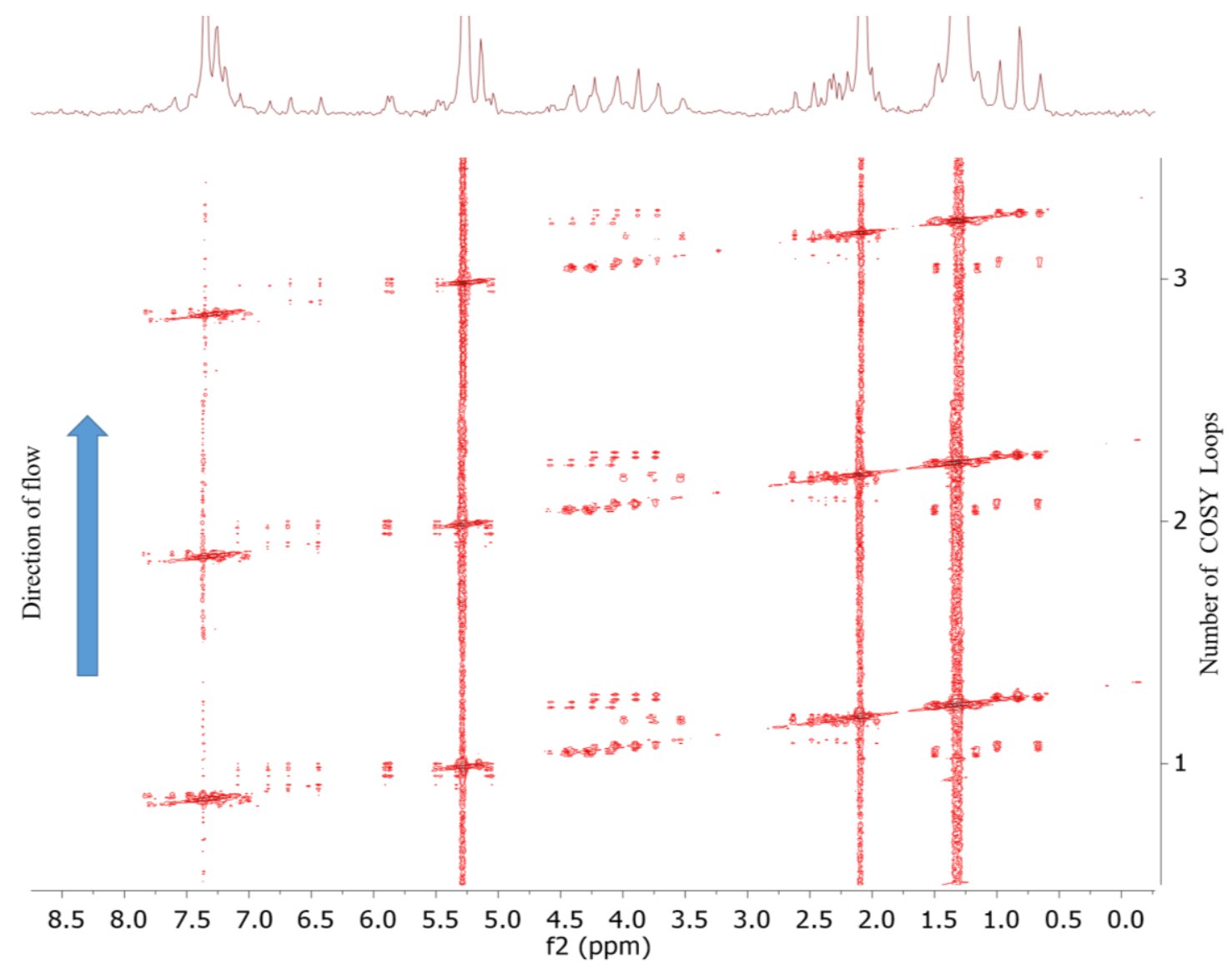

Figure S13. COSY contours acquired by inline monitoring of cyclopropanation steady state of $\mathbf{4}$ using the LEY-2D-COSY loop.

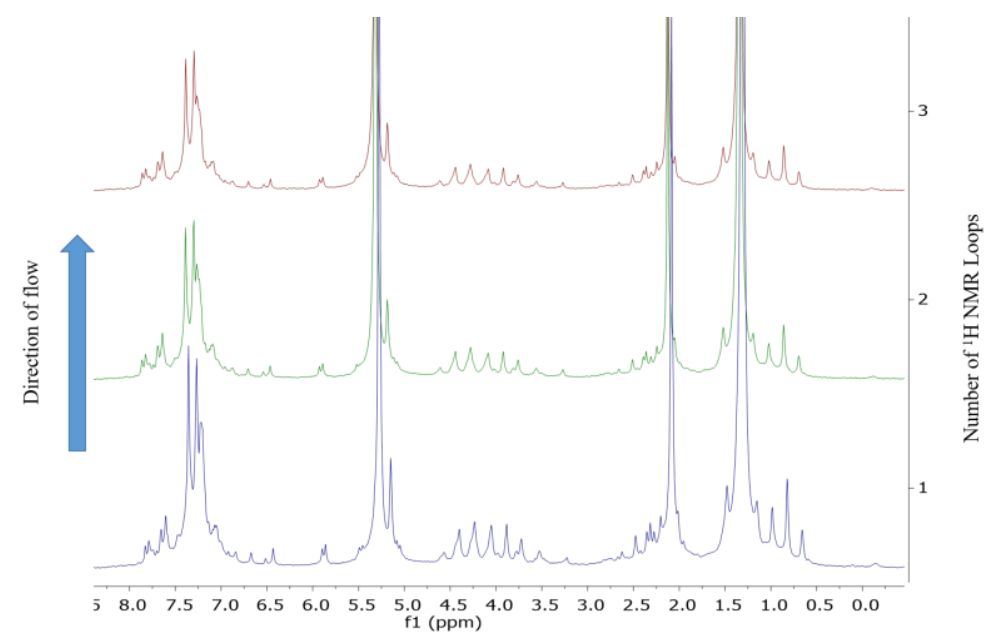

Figure S14. ${ }^{1} \mathrm{H}$ NMR acquired by inline monitoring of cyclopropanation steady state of 4 using the LEY2D-COSY loop. 


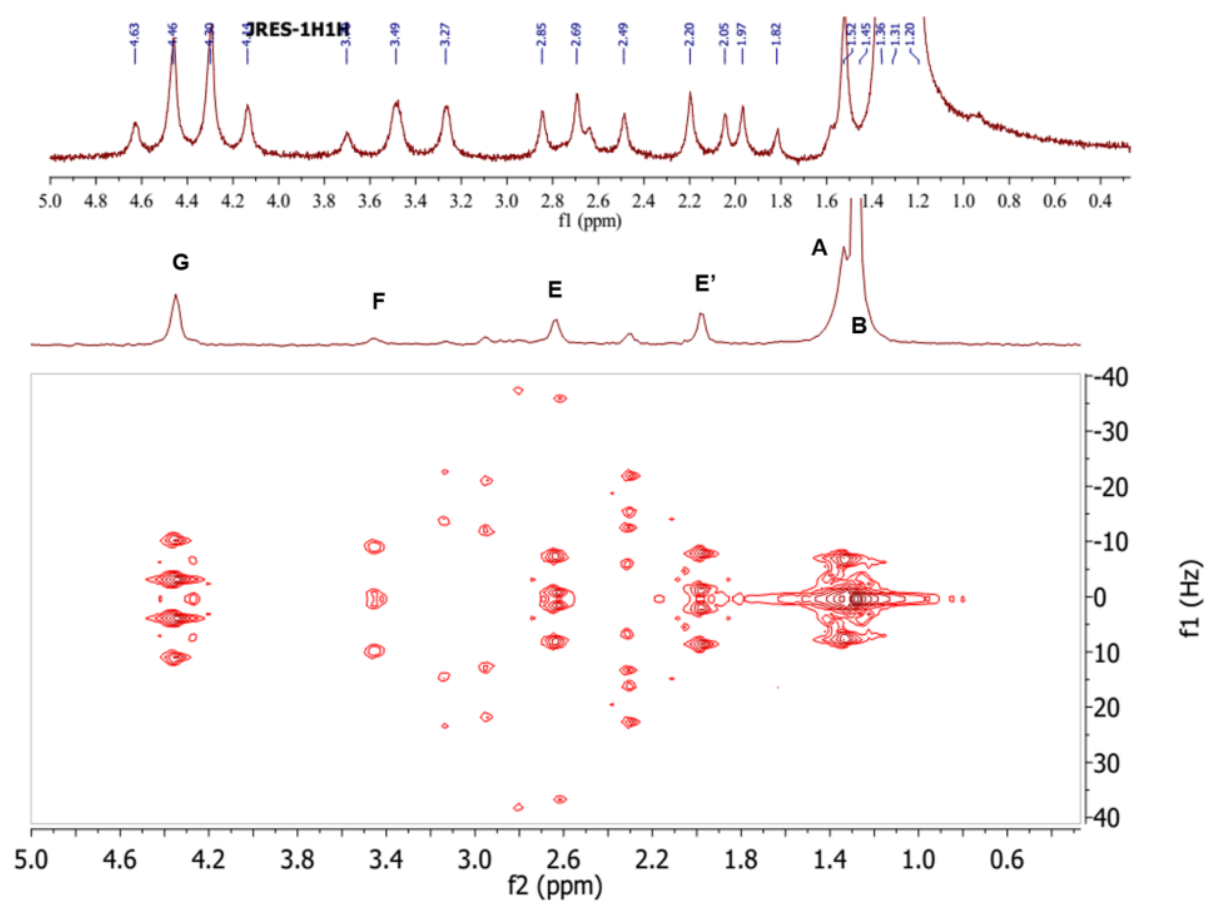

Figure S15. 2D ${ }^{1} \mathrm{H} J$-RES spectrum of isolated $[Z]$ 1-nitro-1-cyclopropylcarboxylate 5.

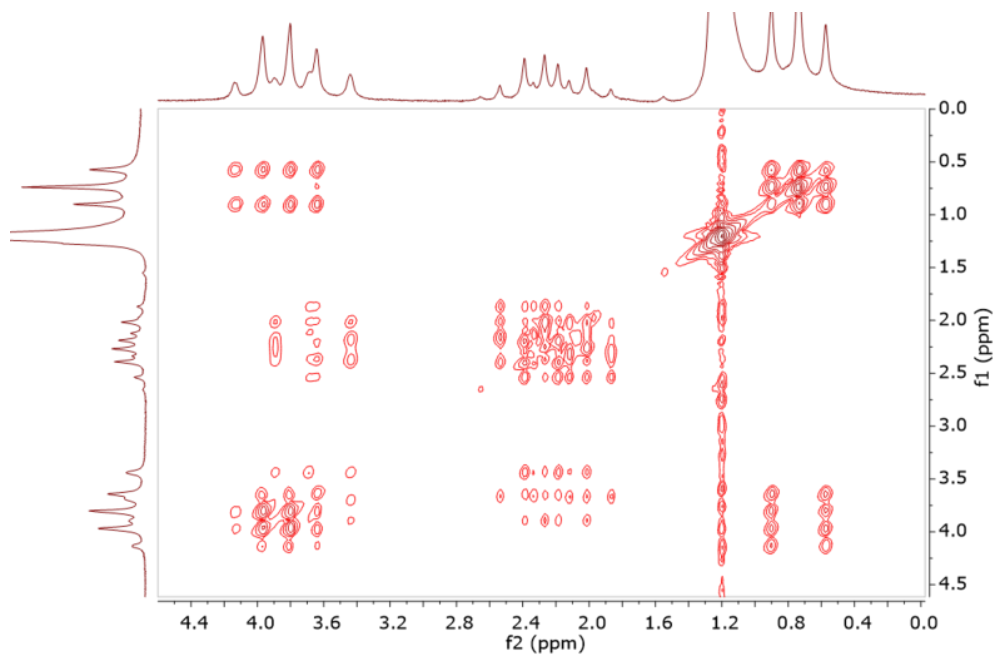

Figure S16.2D H-H COSY contour of isolated $[E]$ 1-nitro-1-cyclopropylcarboxylate 5. 


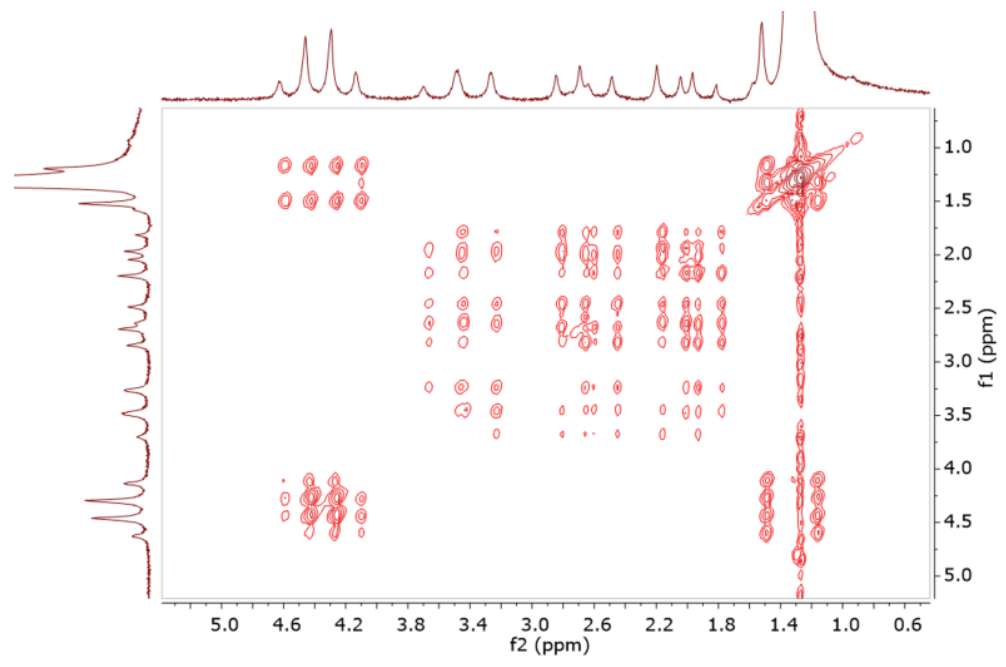

Figure S17.2D H-H COSY contour of isolated [Z] 1-nitro-1-cyclopropylcarboxylate 5. 


\section{Advion Benchtop Mass Spectroscopy:}

Reaction monitoring carried using Advion(C) Expression Compact Mass Spectroscopy. A single quadrupole mass analyzer with atmospheric pressure interface, provides APCI (atmospheric pressure chemical ionization) capabilities with sequential +ve and -ve polarity switching. ${ }^{7}$ Typical APCI parameters: Ion source: Capillary Temp: $250{ }^{\circ} \mathrm{C}$, Capillary Voltage $180 \mathrm{~V}$, APCI gas $\left(\mathrm{N}_{2}\right)$ Temp: $250^{\circ} \mathrm{C}$, Corona discharge: $6 \mathrm{uA}$.
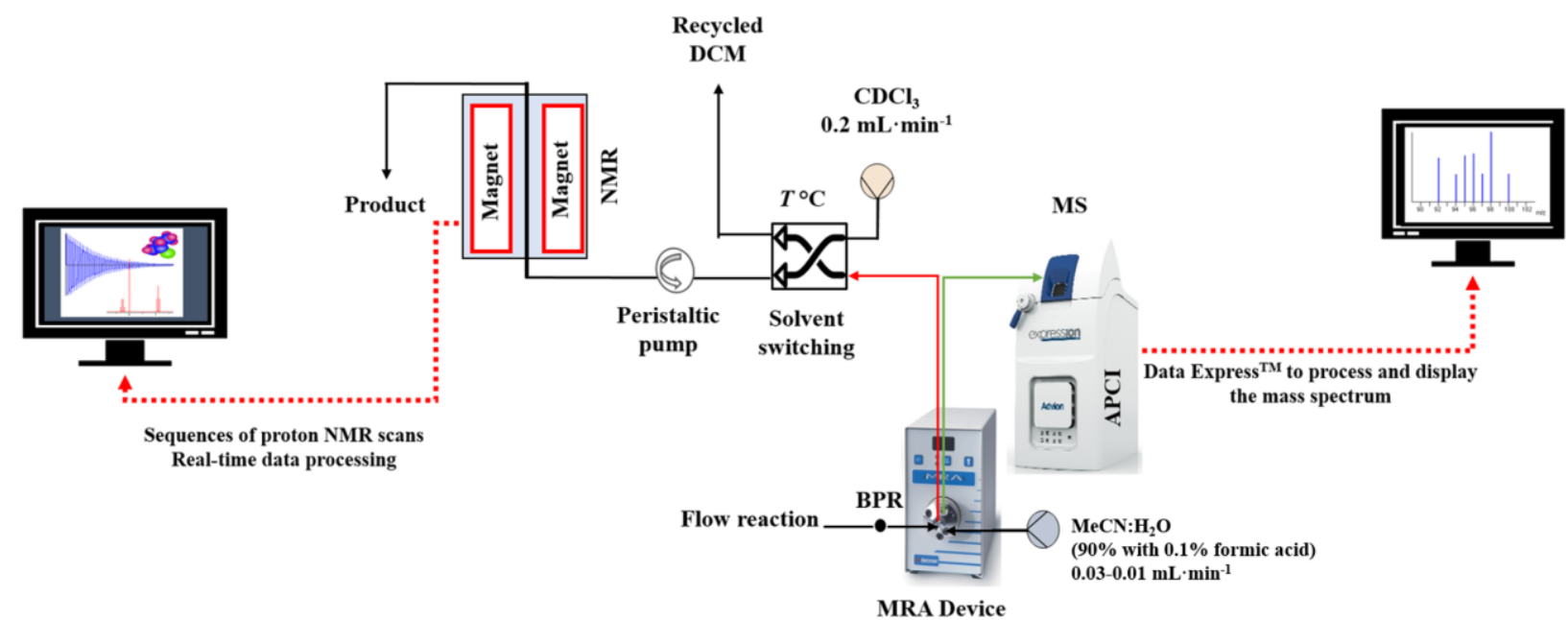

Figure S18. Configuration of the CMS-NMR for multiple reaction monitoring technique. 

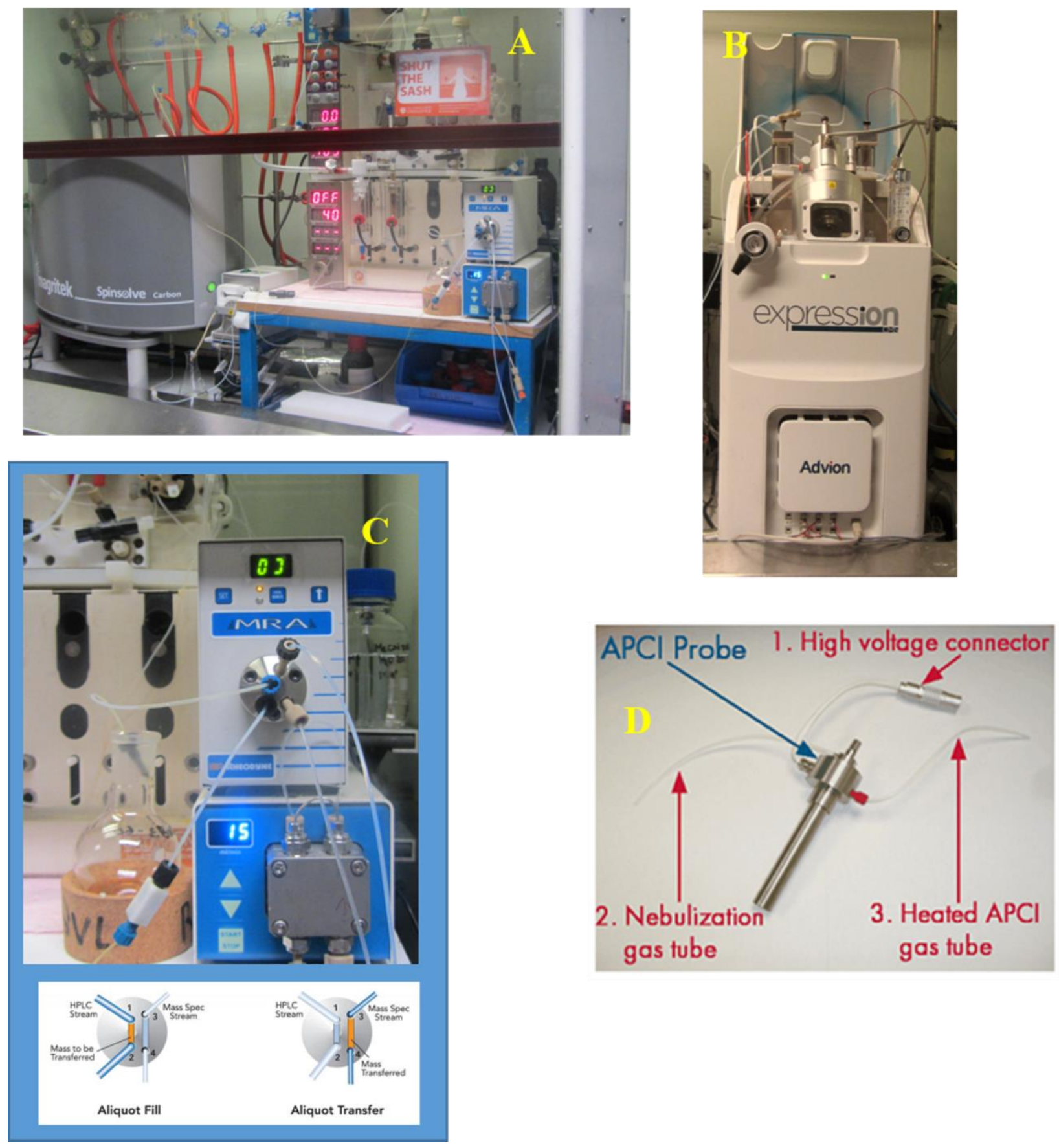

Figure S19. (A) NMR coupled with MS via the $M A^{\odot}$ valve: (B) Advion compact Mass Spectrometry with APCI detector: (C) MRA valve ${ }^{8}$ used for sampling of the flow reaction stream into the MS while diluted and carried using $90 \% \mathrm{MeCN}: \mathrm{H}_{2} \mathrm{O}$ solvent: (D) APCI detector. 

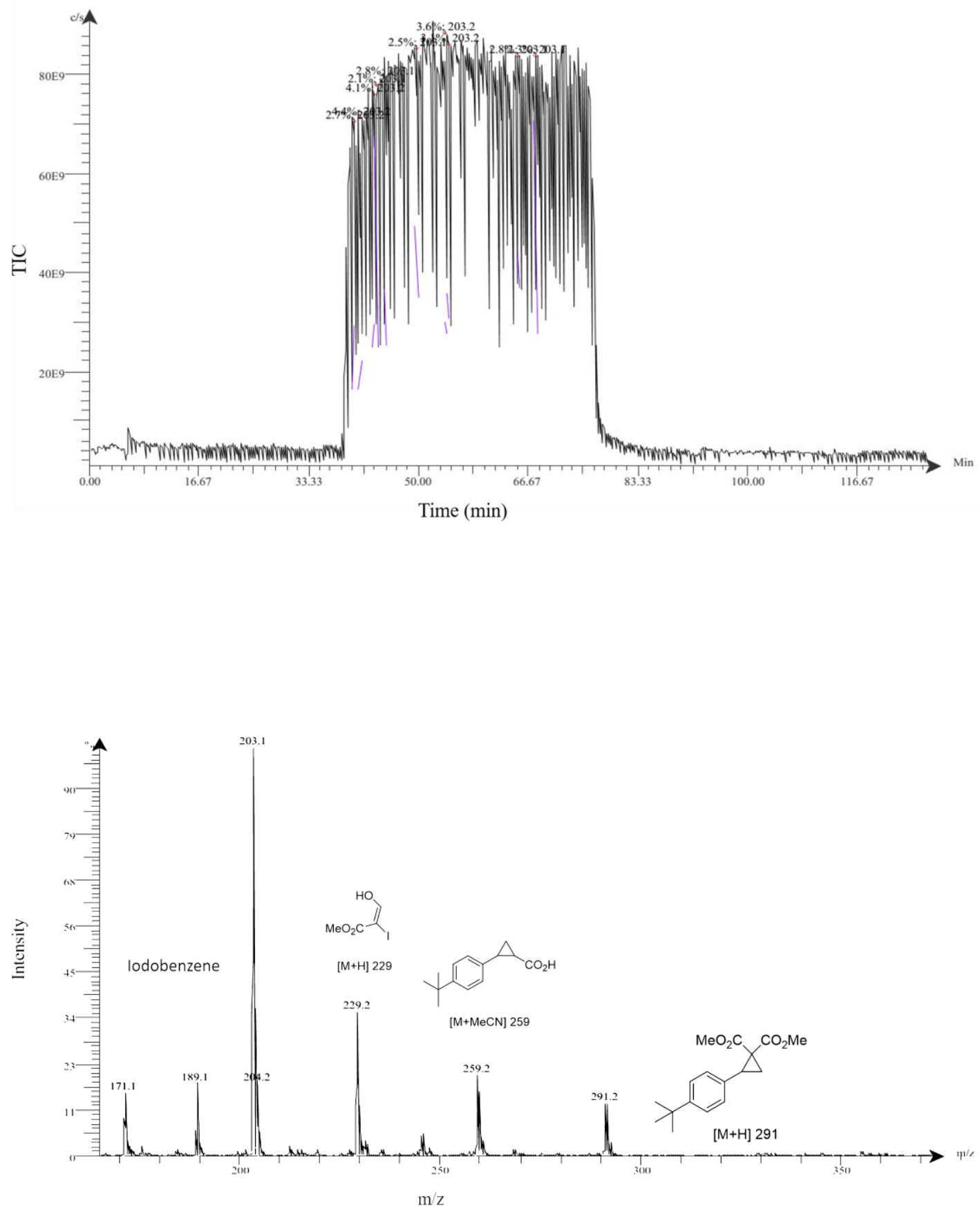

Figure S20. MS spectra (bottom) and total ion count profile (top) of the cyclopropanation reaction of phenyl iodonium ylide 6 . 


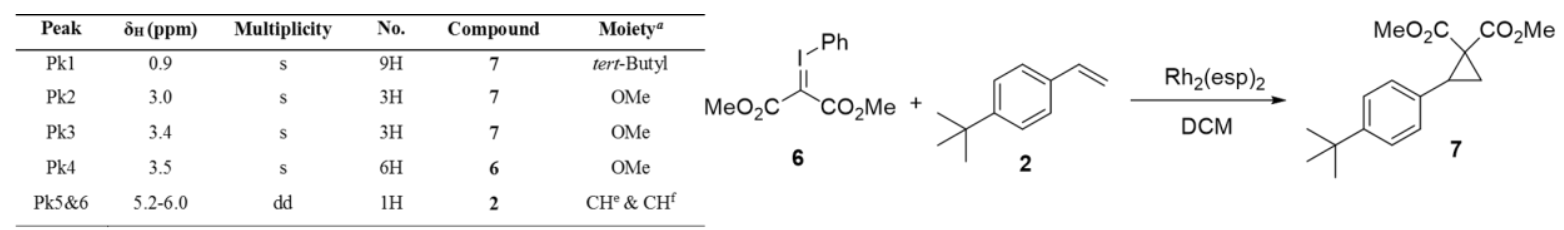

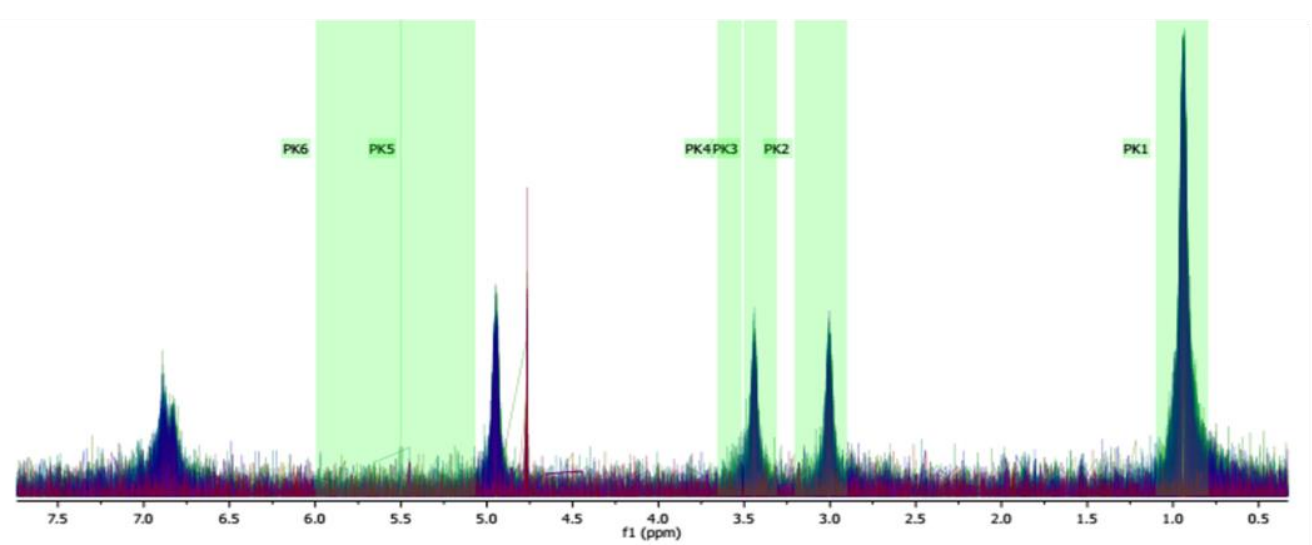

Figure S21. ${ }^{1} \mathrm{H}$ NMR spectrum in $\mathrm{DCM} / \mathrm{CDCl}_{3}$ of the cyclopropanation reaction of phenyl iodonium

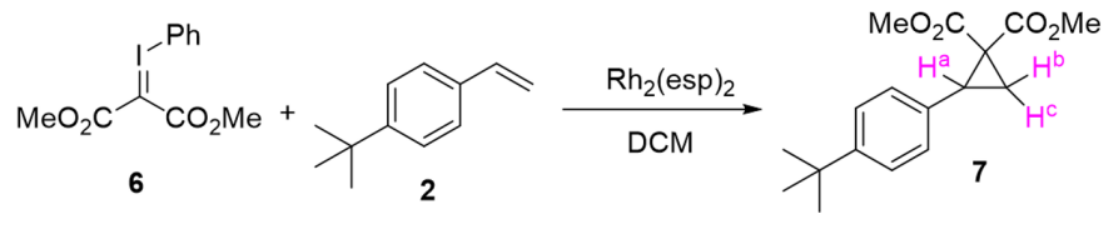

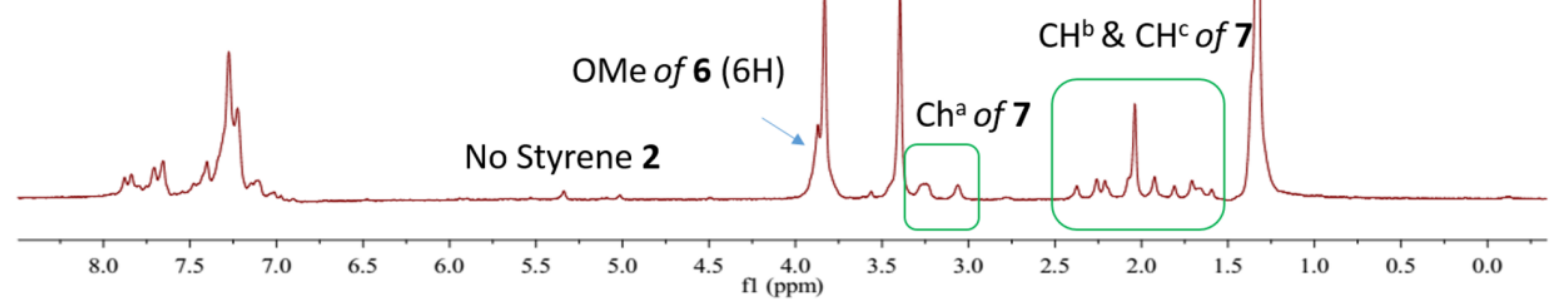

ylide 6. 
Figure S22. ${ }^{1} \mathrm{H}$ NMR spectrum of the collected crude mixture of the cyclopropanation reaction of

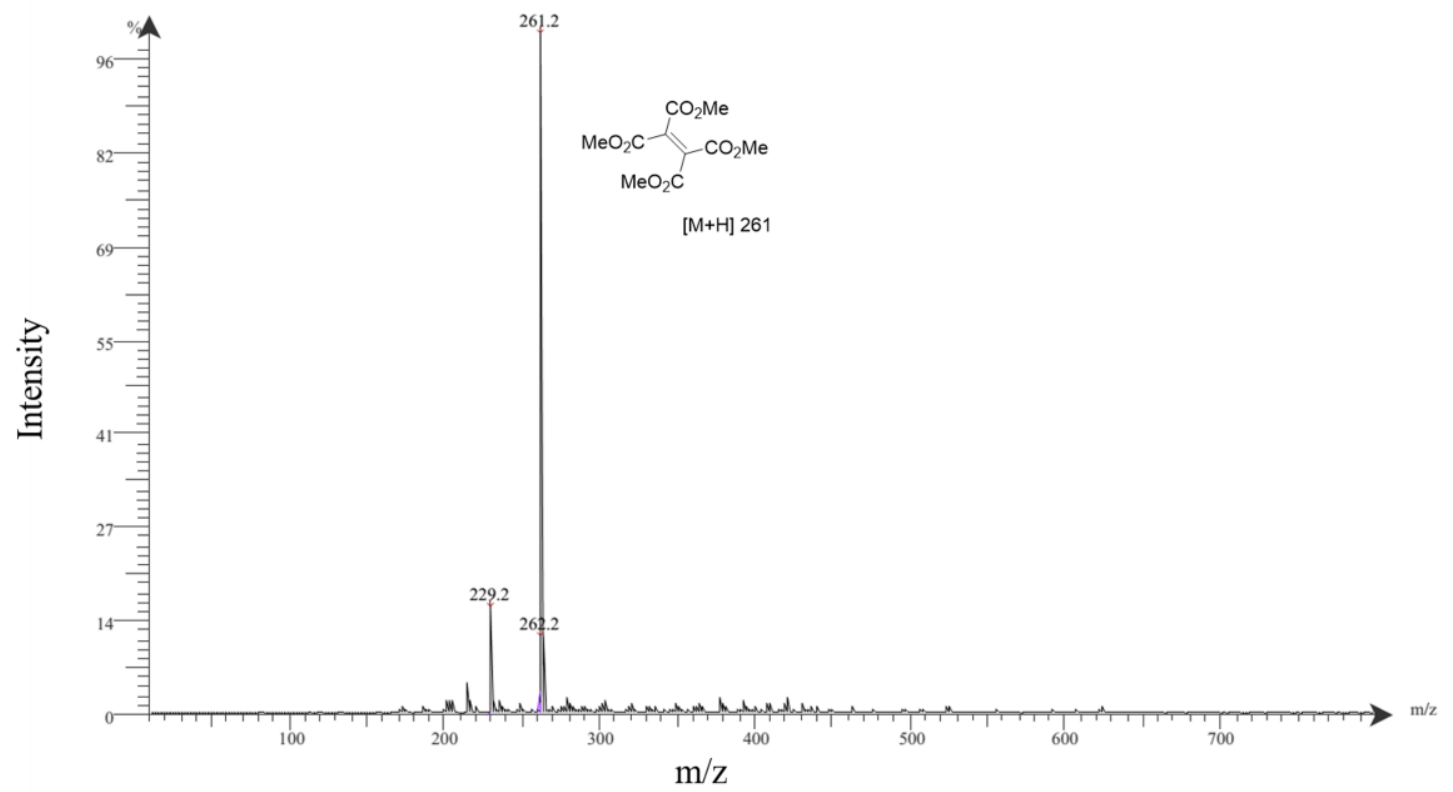

phenyl iodonium ylide $\mathbf{6}$ in $\mathrm{CDCl}_{3}$.

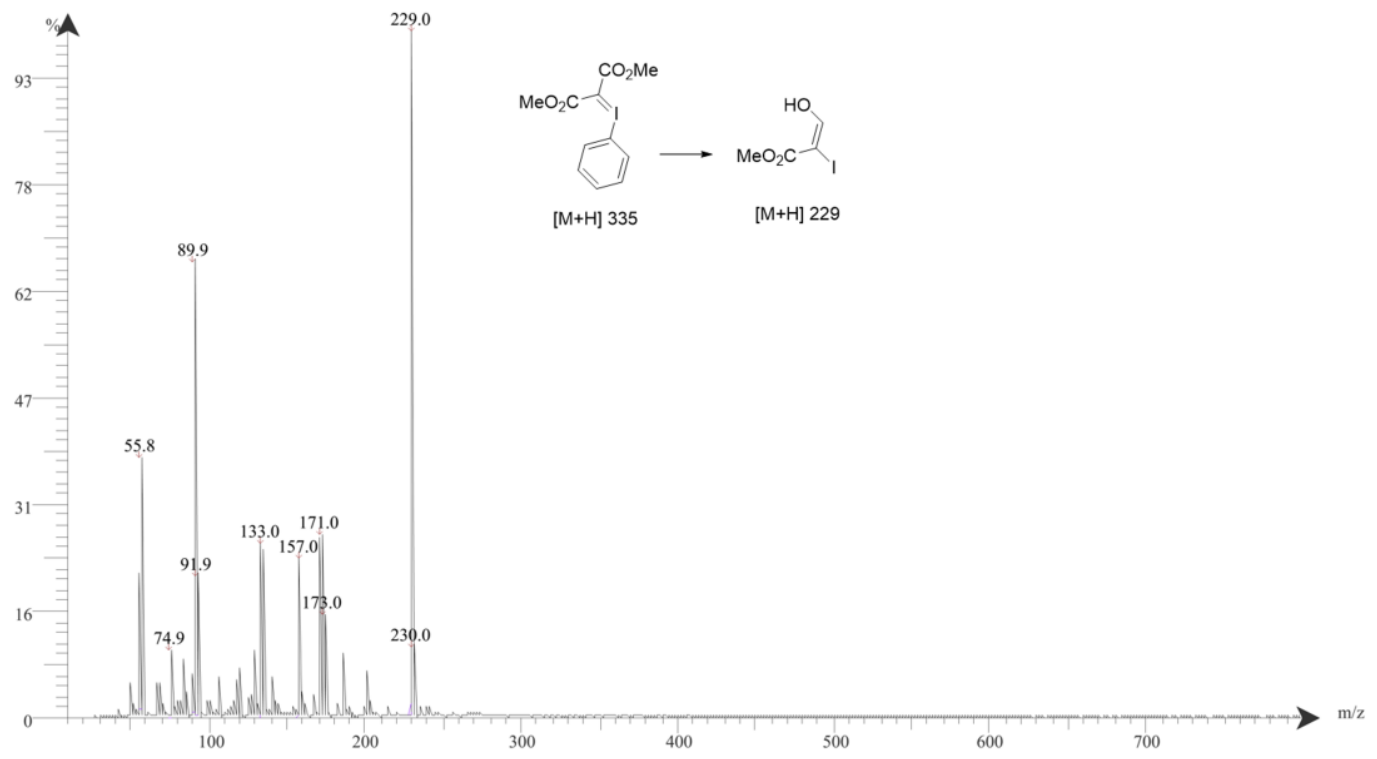

Figure S23. MS spectra of decomposition of phenyl iodonium ylide 6 with $\mathrm{Rh}_{2}(\mathrm{esp})_{2}$ in $\mathrm{DCM}$ under ambient conditions. This control reaction shows the formation of the dimer at m/z $261[\mathrm{M}+\mathrm{H}]$. 
Figure S24. MS spectra phenyl iodonium ylide 6 in DCM under APCI conditions. Only ylide 6 fragment found at $\mathrm{m} / \mathrm{z} 229[\mathrm{M}+\mathrm{H}]$.

\section{Experimental and Analysis:}

Material and Methods: All reagents were used as purchased without further purification, this includes; 1, 2-dichloroethane anhydrous solvent, while dichloromethane and acetonitrile solvents were dried over calcium hydride and distilled prior to use. ${ }^{1} \mathrm{H}$ NMR and ${ }^{13} \mathrm{C}$ NMR spectra of all obtained cyclopropane adducts after purification were recorded using the $600 \mathrm{MHz}$ on Bruker Avance $600 \mathrm{BBI}$ spectrometer. The chemical shifts were reported in ppm with the solvent resonance as the internal standard of $\mathrm{CHCl}_{3}$ at $7.26 \mathrm{ppm} .{ }^{1} \mathrm{H} \mathrm{NMR}$ data are reported as follows: chemical shift, integration, multiplicity $(\mathrm{s}=$ singlet, $\mathrm{d}=$ doublet, $\mathrm{t}=$ triplet, $\mathrm{q}=$ quartet, $\mathrm{dd}=$ doublet of doublets, $\mathrm{m}=$ multiplet), and coupling constant $J$ in Hz. High-resolution mass determination were recorded on LCT premier TOF mass spectrometer, features Z-spay source with ESI, using $50 \% \mathrm{MeCN}: \mathrm{H}_{2} \mathrm{O}(0.25 \%$ Formic acid). Purification of cyclopropane adducts were performed on SiliCycle SiliaSep ${ }^{\mathrm{TM}} 40-63 \mathrm{~mm} 60 \AA$ flash cartridges using an automated Biotage $^{\mathrm{TM}}$ flash chromatography coupled with UV detector at $254 \mathrm{~nm}$ and $280 \mathrm{~nm}$. Thin layer chromatography (TLC) was performed on glass plates pre-coated with silica (Merck DCKieselgel 60 F254), developed and visualized by UV and potassium permanganate dip.

2-(4-(tert-Butyl)-phenyl) cyclopropane-1,1-dicarbonitrile (3): ${ }^{1} \mathrm{H} \mathrm{NMR}\left(600 \mathrm{MHz}, \mathrm{CDCl}_{3}\right): \delta$ 1.33 (s, 9H), 2.22-2.25 (m, 2H), 3.26-3.29 (t, $J=9.1 \mathrm{~Hz}, 1 \mathrm{H}), 7.23-7.24(\mathrm{~d}, J=8.3 \mathrm{~Hz}, 2 \mathrm{H})$, 7.44-7.45 (d, $J=8.4 \mathrm{~Hz}, 2 \mathrm{H}) .{ }^{13} \mathrm{C}$ NMR $\left(150 \mathrm{MHz}, \mathrm{CDCl}_{3}\right) \delta 152.69,127.99,127.46,126.07$, $115.45,113.15,35.01,34.73,31.19,22.48,7.16 .^{9}$

Ethyl 2-(4-tert-butylphenyl)-1-nitrocyclopropanecarboxylate (5): [E]-isomer: ${ }^{1} \mathrm{H}$ NMR (600 $\left.\mathrm{MHz}, \mathrm{CDCl}_{3}\right): \delta$ 0.80-0.85 (t, $\left.J=7.1 \mathrm{~Hz}, 3 \mathrm{H}\right), 1.29(\mathrm{~s}, 9 \mathrm{H}), 2.16-2.21(\mathrm{dd}, J=10.7,6.6 \mathrm{~Hz}, 1 \mathrm{H})$, 2.41-2.46 (dd, $J=9.1,6.6 \mathrm{~Hz}, 1 \mathrm{H}), 3.72-3.77(\mathrm{~m}, 1 \mathrm{H}), 3.88-4.04(\mathrm{~m}, 2 \mathrm{H}), 7.12-7.15(\mathrm{~d}, J=8.3$ $\mathrm{Hz}, 2 \mathrm{H}), 7.30-7.37$ (d, $J=8.4 \mathrm{~Hz}, 2 \mathrm{H}) .{ }^{13} \mathrm{C} \mathrm{NMR}\left(150 \mathrm{MHz}, \mathrm{CDCl}_{3}\right) \delta 161.96,151.38,128.96$, $128.15,125.37,71.71,62.37,34.54,33.91,31.21,20.73,13.35$. HRMS (ESI) Calcd. for C16H21NO4 ([M+H]+): 292.15, Found 292.15: [Z]-isomer: ${ }^{1} \mathrm{H}$ NMR $\left(600 \mathrm{MHz}, \mathrm{CDCl}_{3}\right): \delta 1.30$ $(\mathrm{s}, 9 \mathrm{H}), 1.34-1.37(\mathrm{t}, J=7.1 \mathrm{~Hz}, 3 \mathrm{H}), 2.00-2.04(\mathrm{dd}, J=9.9,6.9 \mathrm{~Hz}, 1 \mathrm{H}), 2.64-2.68(\mathrm{dd}, J=9.2$, $6.9 \mathrm{~Hz}, 1 \mathrm{H}), 3.43-3.48(\mathrm{t}, J=9.6 \mathrm{~Hz}, 1 \mathrm{H}), 4.31-4.41(\mathrm{~m}, 2 \mathrm{H}), 7.14-7.17(\mathrm{~d}, J=8.3 \mathrm{~Hz}, 2 \mathrm{H})$, 7.32-7.36 (d, $J=8.4 \mathrm{~Hz}, 2 \mathrm{H}) .{ }^{13} \mathrm{C}$ NMR $\left(150 \mathrm{MHz}, \mathrm{CDCl}_{3}\right) \delta 165.40,151.56,128.33,127.99$, $125.63,72.63,63.14,34.55,33.48,31.22,20.08,13.98 .^{10}$

Bis-(methoxycarbonyl)-(phenyliodinio)-methanide (6): ${ }^{1} \mathrm{H}$ NMR (600 MHz, DMSO- $\left.d_{6}\right) \delta$ 7.75-7.70 (d, $J=7.9 \mathrm{~Hz}, 2 \mathrm{H}), 7.55-7.50(\mathrm{t}, J=7.3 \mathrm{~Hz}, 1 \mathrm{H}), 7.45-7.40$ (t, $J=7.6 \mathrm{~Hz}, 2 \mathrm{H}), 3.5$ (s, $6 \mathrm{H}) .{ }^{13} \mathrm{C}$ NMR $\left(150 \mathrm{MHz}, \mathrm{DMSO}-d_{6}\right) \delta 170.78,136.28,136.08,135.87,135.37,132.90,121.21$, $56.20 .^{11}$

Dimethyl 2-(4-tert-butylphenyl)cyclopropane-1,1-dicarboxylate (7): ${ }^{1} \mathrm{H}$ NMR (600 $\mathrm{MHz}$, CDCl3): $\delta 1.29$ (s, 9H), 1.70-1.75 (dd, $J=9.3,5.1 \mathrm{~Hz}, 1 \mathrm{H}), 2.16-2.20(\mathrm{dd}, J=8.0,5.1 \mathrm{~Hz}, 1 \mathrm{H})$, 
3.18-3.22 (t, $J=8.6 \mathrm{~Hz}, 1 \mathrm{H}), 3.34$ (s, $3 \mathrm{H}), 3.78(\mathrm{~s}, 3 \mathrm{H}), 7.10-7.14(\mathrm{~d}, J=8.4 \mathrm{~Hz}, 2 \mathrm{H}), 7.27-7.31$ $(\mathrm{d}, J=8.4 \mathrm{~Hz}, 2 \mathrm{H}) .{ }^{13} \mathrm{C} \mathrm{NMR}\left(150 \mathrm{MHz}, \mathrm{CDCl}_{3}\right) \delta 170.27,167.09,150.30,131.47,128.10$, $127.89,127.59,125.51,125.18,125.01,124.81,124.28,52.69,52.13,52.07,37.16$, $34.43,32.86,32.28,31.26,19.29 .^{12}$

2-Methyl-2-phenylcyclopropane-1,1-dicarbonitrile (9): ${ }^{1} \mathrm{H} \mathrm{NMR}\left(600 \mathrm{MHz}, \mathrm{CDCl}_{3}\right): \delta=1.79$ (s, 3H), 1.97-1.99 (d, $J=6.0 \mathrm{~Hz}, 1 \mathrm{H}), 2.33-2.35(\mathrm{~d}, J=6.0 \mathrm{~Hz}, 1 \mathrm{H}), 7.36-7.44(\mathrm{~m}, 5 \mathrm{H}) .{ }^{13} \mathrm{C}$ NMR $\left(150 \mathrm{MHz}, \mathrm{CDCl}_{3}\right): \delta 136.81,129.30,129.17,128.19,114.39,113.93,40.31,29.81,24.35$, $11.55 .^{13}$

2-(2-Fluorophenyl)-cyclopropane-1,1-dicarbnitrile (10): ${ }^{1} \mathrm{H} \mathrm{NMR}\left(600 \mathrm{MHz}, \mathrm{CDCl}_{3}\right): \delta 2.22-$ $2.36(\mathrm{~m}, 2 \mathrm{H}), 3.34-3.37(\mathrm{t}, J=9.1 \mathrm{~Hz}, 1 \mathrm{H}), 7.13-7.16(\mathrm{t}, J=6.9 \mathrm{~Hz}, 1 \mathrm{H}), 7.14-7.22(\mathrm{~m}, 2 \mathrm{H})$, 7.41-7.45 (m, 1H). ${ }^{13} \mathrm{C}$ NMR $\left(150 \mathrm{MHz}, \mathrm{CDCl}_{3}\right) \delta 163.05,161.39,132.75,131.50,131.44$, $128.70,128.68,124.64,124.62,118.70,118.61,116.17,116.03,114.89,112.84,29.28,29.25$, $21.99,21.98,6.62 .^{14}$

Ethyl 2-(4-fluorophenyl)-1-nitrocyclopropanecarboxylate (11): [E]-isomer: ${ }^{1} \mathrm{H}$ NMR (600 $\left.\mathrm{MHz}, \mathrm{CDCl}_{3}\right): \delta=0.95-0.98(\mathrm{t}, J=7.2 \mathrm{~Hz}, 3 \mathrm{H}), 2.19-2.22(\mathrm{dd}, J=10.8,6.7 \mathrm{~Hz}, 1 \mathrm{H}), 2.39-2.42$ (dd, $J=9.1,6.7 \mathrm{~Hz}, 1 \mathrm{H}), 3.71-3.76(\mathrm{t}, J=9.9 \mathrm{~Hz}, 1 \mathrm{H}), 3.97-4.03(\mathrm{q}, J=7.1 \mathrm{~Hz}, 2 \mathrm{H}), 6.99-7.02$ $(\mathrm{t}, J=8.6 \mathrm{~Hz}, 2 \mathrm{H}), 7.19-7.22(\mathrm{dd}, J=8.5,5.3 \mathrm{~Hz}, 2 \mathrm{H}) .{ }^{13} \mathrm{C} \mathrm{NMR}\left(151 \mathrm{MHz}, \mathrm{CDCl}_{3}\right) \delta 163.37$, $161.83,161.73,130.30,130.24,127.87,127.85,115.54,115.40,71.53,62.61,33.19,20.74$, 13.55. HRMS (ESI) Calcd. for C12H12FNO ([M+H]+): 254.08, Found 254.08. ${ }^{15}$

Ethyl 2-methyl-1-nitro-2-phenylcyclopropanecarboxylate (12): [E]-isomer: ${ }^{1} \mathrm{H}$ NMR $(600$ $\mathrm{MHz}, \mathrm{CDCl}_{3}$ ): $\delta=0.87-0.90$ (t, $\left.J=7.1 \mathrm{~Hz}, 3 \mathrm{H}\right), 1.54(\mathrm{~s}, 3 \mathrm{H}), 2.15-2.17(\mathrm{~d}, J=6.8 \mathrm{~Hz}, 1 \mathrm{H})$, 2.42-2.44 (d, $J=6.8 \mathrm{~Hz}, 1 \mathrm{H}), 3.87-3.97(\mathrm{~m}, 2 \mathrm{H}), 7.26-7.35(\mathrm{~m}, 5 \mathrm{H}) .{ }^{13} \mathrm{C}$ NMR $(150 \mathrm{MHz}$, $\left.\mathrm{CDCl}_{3}\right) \delta 163.55,139.06,133.09,128.59,128.56,128.30,127.74,127.66,76.15,62.44,39.11$, $26.60,26.29,24.91,13.43 .^{16}$

Ethyl 1-nitro-2-(3-nitrophenyl)cyclopropane-carboxylate (13): [E]-isomer: ${ }^{1} \mathrm{H}$ NMR $(600$ $\left.\mathrm{MHz}, \mathrm{CDCl}_{3}\right): \delta 0.98-1.01(\mathrm{t}, J=7.1 \mathrm{~Hz}, 3 \mathrm{H}), 2.29-2.33(\mathrm{dd}, J=10.7,6.9 \mathrm{~Hz}, 1 \mathrm{H}), 2.48-2.52$ $(\mathrm{dd}, J=9.1,6.9 \mathrm{~Hz}, 1 \mathrm{H}), 3.81-3.85(\mathrm{~m}, 1 \mathrm{H}), 4.01-4.06(\mathrm{q}, J=7.1 \mathrm{~Hz}, 2 \mathrm{H}), 7.51-7.56(\mathrm{t}, J=7.9$ $\mathrm{Hz}, 1 \mathrm{H}), 7.59-7.62(\mathrm{~d}, J=7.7 \mathrm{~Hz}, 1 \mathrm{H}), 8.11(\mathrm{~s}, 1 \mathrm{H}), 8.17-8.19(\mathrm{~m}, 1 \mathrm{H}) .{ }^{13} \mathrm{C}$ NMR $(150 \mathrm{MHz}$, $\left.\mathrm{CDCl}_{3}\right) \delta 161.57,148.24,134.82,134.61,134.42,129.60,128.58,123.50,123.28,71.30,62.99$, $32.69,20.52,13.61 .^{17}$

Dimethyl 2-(4-fluorophenyl)cyclopropane-1,1-dicarboxylate (14): ${ }^{1} \mathrm{H}$ NMR (600 $\mathrm{MHz}$, CDCl3): $\delta 1.73-1.75(\mathrm{dd}, J=9.3,5.2 \mathrm{~Hz}, 1 \mathrm{H}), 2.14-2.16(\mathrm{dd}, J=8.0,5.2 \mathrm{~Hz}, 1 \mathrm{H}), 3.18-3.21(\mathrm{t}, J$ $=8.6 \mathrm{~Hz}, 1 \mathrm{H}), 3.39(\mathrm{~s}, 3 \mathrm{H}), 3.79(\mathrm{~s}, 3 \mathrm{H}), 6.91-7.01(\mathrm{~m}, 2 \mathrm{H}), 7.12-7.22(\mathrm{~m}, 2 \mathrm{H}) .{ }^{13} \mathrm{C}$ NMR $(150$ $\left.\mathrm{MHz}, \mathrm{CDCl}_{3}\right) \delta 170.07,166.92,162.93,161.30,130.30,130.28,130.15,130.10,115.16,115.01$, $52.81,52.27,37.05,31.71,19.20 .^{18}$ 


\section{References:}

${ }^{1}$ Spinsolve, Magriteck, http://www.magritek.com/products/spinsolve/ (last visited 15/05/2016).

${ }^{2}$ Upchurch Scientific (Idex Health and Science), http://webstore.idex-hs.com / (last visited 15/05/2016).

${ }^{3}$ Vapourtec Ltd, http://www.vapourtec.co.uk (last visited 15/05/2016).

${ }^{4}$ Omnifit (Diba), http://www.omnifit.com/ (last visited 15/05/2016).

${ }^{5}$ Cole-Parmer Instrument Co. Ltd., http://www.coleparmer.co.uk/ (last visited 15/05/2016).

${ }^{6}$ Swagelok Company, http://www.swagelok.co.uk/landingpages/index-uk.htm, (last visited 15/05/2016).

${ }^{7}$ Advion, http://www.advion.com/products/expression/high-performance-compact-massspectrometer/, (last visited 15/05/2016).

${ }^{8}$ Rheodyne MRA, http://kinesis.co.uk/knowledgebase/rheodyne-mra, (last visited 15/05/2016).

9 Lin, S.; Li, M.; Dong, Z.; Liang, F.; Zhang, J. Org. Biomol. Chem. 2014, 12, 1341-1350.

${ }^{10}$ Zhu, S.; Perman, J. A.; Zhang, X. P. Angew. Chem. Int. Ed. 2008, 47, 8460-8463.

${ }^{11}$ Goudreau, S. R.; Marcoux, D.; Charette, A. B. Org. Synth. 2010, 87, 115-125.

${ }^{12}$ Perreault, C.; Goudreau, S. R.; Zimmer, L. E.; Charette, A. B. Org. Lett. 2008, 10(5), 689-692.

${ }^{13}$ Lin, S.; Li, M.; Dong, Z.; Liang, F.; Zhang, J. Org. Biomol. Chem. 2014, 12, 1341-1350.

${ }^{14}$ Li, J.; Liu, Y-C.; Deng, J-G.; Li, X-Z.; Cui, X.; Li, Z. Tetrahedron: Asymmetry 2000, 11, 2677-2682.

${ }^{15}$ Zhu, S.; Perman, J. A.; Zhang, X. P. Angew. Chem. Int. Ed. 2008, 47, 8460-8463.

${ }^{16}$ Zhu, S.; Perman, J. A.; Zhang, X. P. Angew. Chem. Int. Ed. 2008, 47, 8460-8463.

${ }^{17}$ Gopinath, P.; Chandrakala, R. N.; Chandrasekaran, S. Synthesis 2015, 47, 1488-1498.

${ }^{18}$ Perreault, C.; Goudreau, S. R.; Zimmer, L. E.; Charette, A. B. Org. Lett. 2008, 10(5), 689-692. 